\title{
The Effects and Underlying Mechanisms of Hepatitis B Virus X Gene Mutants on the Development of Hepatocellular Carcinoma
}

\section{Rui Pu}

The Second Military Medical University

\section{Wenbin Liu}

Second Military Medical University

\section{Xinyu Zhou}

Second Military Medical University

\section{Xiaomei Hou}

Second Military Medical University

\section{Shiliang Cai}

Second Military Medical University

Xi Chen

Second Military Medical University

Liping Chen

Second Military Medical University

Jianfeng Wu

Second Military Medical University

\section{Fan Yang}

Second Military Medical University

\section{Xiaojie Tan}

Second Military Medical University

\section{Jianhua Yin}

Second Military Medical University

\section{Xin Wang}

Stem Cell Institute, University of Minnesota

Guangwen Cao ( $\sim$ gcao@smmu.edu.cn )

The Second Military Medical University https://orcid.org/0000-0002-8094-1278

\section{Research}

Keywords: Hepatitis B virus, mutation, hepatocarcinogenesis, PAI1, CDC20 
Posted Date: August 31st, 2021

DOl: https://doi.org/10.21203/rs.3.rs-818602/v1

License: (c) (i) This work is licensed under a Creative Commons Attribution 4.0 International License. Read Full License

Version of Record: A version of this preprint was published at Frontiers in Oncology on February 10th, 2022. See the published version at https://doi.org/10.3389/fonc.2022.836517. 


\section{Abstract}

Background: We aimed to elucidate the mechanism by which hepatitis $B$ virus $\mathrm{X}(\mathrm{HBx})$ gene mutations increase the risk of hepatocellular carcinoma (HCC) and identify novel therapeutic targets.

Methods: Wild-type and four HBx mutants (M1, A1762T/G1764A; M2,

T1674G+T1753C+A1762T/G1764A; M3, C1653T+T1674G+A1762T/G1764A; Ct-HBx, carboxylic acidterminal truncated $\mathrm{HBx}$ ) were delivered into the livers of fumarylacetoacetate hydrolase-deficient mice by using the Sleeping Beauty $(S B)$ transposon system, respectively. Seven liver tissues and seven tumor tissues of the $S B$ mouse models were subjected to HBV-capture sequencing. Three liver tissues from WT$\mathrm{HBx}$ mice, three tumor tissues from M3-HBx mice, and three tumor tissues from $\mathrm{Ct}-\mathrm{HBx}$ mice were subjected to cDNA microarray analysis. HeLa cells stably expressing WT-HBx and the four HBx mutants were also subjected to cDNA microarray assay.

Results: The incidence of HCC was higher in the mice injected with M3-HBx or Ct-HBx. M3-HBx had a stronger capacity of upregulating inflammatory cytokines than other $\mathrm{HBx}$ variants. HBV-capture sequencing showed that the HBx fragments were mainly integrated into intergenic and intron regions. No significant difference was observed in the number of insertion sites between tumors and liver tissues. Ectopic expression of the HBx mutants, especially M3-HBx and Ct-HBx, significantly increased cell proliferation and the S phase proportion of HepG2 and HeLa cells, compared to WT-HBx. Liver tissues of the $S B$ mice and the transfected cells were subjected to CDNA microarray analysis. Plasminogen activator inhibitor-1 (PAl1) and cell division cycle 20 (CDC20) were identified as novel effectors. M3-HBx and Ct$\mathrm{HBx}$ significantly upregulated the expression of PAl1 and CDC20 in HepG2 and HeLa cells as well as the livers of the $S B$ mice. PAl1 silencing attenuated the effect of M3-HBx and Ct-HBx on the growth of HepG2 cells and greatly decreased the growth of HeLa cells with Ct-HBx.

Conclusion: HBx C1653T+T1674G+A1762T/G1764A mutant and Ct-HBx promote carcinogenesis via upregulating PAl1 and CDC20. PAl1, an important player bridging the HBx mutants and HCC, should be a promising candidate as a predictive and prognostic biomarker and therapeutic target in HBV-related HCC.

\section{Background}

Primary liver cancer (PLC) was the third leading cause of cancer death worldwide in 2020 and hepatocellular carcinoma (HCC) accounts for $75-85 \%$ of PLC cases [1]. Chronic infection with hepatitis B virus (HBV) is the leading cause, contributing to $44-55 \%$ of global HCC [2]. Different countries have different rates of the seropositivity for HBV surface antigen (HBsAg) in HCC. Around $50-80 \% \mathrm{HCC}$ cases in China are seropositive for HBsAg [3]. HBV-related HCC (HBV-HCC) is associated with 10-year earlier onset, higher a-fetoprotein (AFP), and more microvascular invasion than HCC caused by other causes [4], indicating that $\mathrm{HBV}$ is more powerful in promoting $\mathrm{HCC}$ development than other etiological factors.

During HBV-induced hepatocarcinogenesis, HBV often evolves via accumulating its mutations adapted to the inflammatory microenvironment and integrating into the human genome $[5,6]$. HBV mutations, 
including A1762T/G1764A, C1653T, T1753V, and T1674G/C, in the core promoter (CP) region of the viral genome are typically the ones that increase the risk of HCC [7-9]. It has been demonstrated that the combination (combo) mutant, rather than $\mathrm{HBx}$ with single or double CP mutations, accelerates cell cycle progression and p21 degradation, possibly via increasing expression of S-phase kinase-associated protein 2 (SKP2) [10]. Gene expression profiling of human hepatocytes infected with HBV genotype F1b, particularly the $\mathrm{CP}$ mutant, indicates more cancer-related signaling pathways compared with other genotypes [8]. HBV integration into the host genome often leads to the truncation of the HBV genome, particularly at the $\mathrm{C}$ terminus of $\mathrm{HBx}$, resulting in the generation of carboxylic acid terminal-truncated $\mathrm{HBV}$ $X$ protein (Ct-HBx) [11]. The HBV X genes encoding Ct-HBX, which are most frequently detected in HBV$\mathrm{HCC}$ samples, significantly increase the aggressiveness of HCC compared to the full-length $\mathrm{X}$ gene, possibly via downregulating thioredoxin-interacting protein, a well-established regulator of glucose metabolism [12]. However, the gene expression profiles of the HBV mutant-integrated liver of animal models with intact immune system, which represents the key molecular event in humans, are not reported. Here, we investigated tumorigenic effects of combo HBx mutations (A1762T/G1764A, C1653T, T1753C, and T1674G), Ct-HBx, and wild-type (WT) HBx on HCC using the Sleeping Beauty (SB) transposon system to deliver WT-HBx and HBx mutants into the livers of fumarylacetoacetate hydrolase (Fah)-deficient mice. The functional genes with similar profiles in the animal model and cell lines were functionally investigated. This study not only helps elucidate the mechanisms by which HBx mutants promote HBV-induced carcinogenesis but also reveals effective therapeutic options for HBV-HCC.

\section{Methods}

\section{Plasmid construction}

HBx fragments (nt. 1374-1838) were amplified from the sera of $10 \mathrm{HBV}$-infected patients included in a previous study [9]. The details for viral DNA extraction, HBV DNA sequencing, and mutation analysis are provided in the Supplementary Materials. We selected four HBx mutants carrying the HCC-risk mutations: M1, A1762T/G1764A alone; M2, A1762T/G1764A + T1674G + T1753C; M3, A1762T/G1764A + C1653T + T1674G, and Ct-HBx (nt. 1374-1733) (Figure S1). WT-HBx and the HBx mutants were linked with flag tags and inserted into ECOR/ restriction sites of the pKT2-FAH-mCa-SB plasmid, a $S B$ transposon vector containing the cDNA of Fah gene, respectively [13]. The constructs were verified by Sanger sequencing. Primers for the amplification and verification are listed in Table S1. The construction of recombinant lentiviruses expressing WT-HBx and HBx mutants is described in the Supplementary Materials.

\section{Cell experiments}

HepG2 and HeLa cell lines were purchased from the Chinese Academy of Sciences (Shanghai, China). Before the experiments, all cell lines were authenticated using the genotyping analysis of short tandem repeat (STR) by Biowing Biotechnology (Shanghai, China). All cell cultures were tested for mycoplasma contamination every three months. The cells with the stable overexpression of $\mathrm{HBx}$ variants were constructed with lentivirus. Details are available in the Supplementary Materials. Small interfering RNAs 
(siRNAs) against cell division cycle 20 (CDC20), plasminogen activator inhibitor-1 (PAl1), and cyclin dependent kinase inhibitor 1A (P21) were synthesized by GenePharma (Shanghai, China) (Table S2). Lipofectamine 3000 kit (Invitrogen, Carlsbad, CA) was applied for siRNA transfection. Gene expression was measured by quantitative reverse transcription PCR (qRT-PCR) and Western blot (Figure S2). Cell proliferation, migration, invasion, cell cycle assay, qRT-PCR, and Western blot are detailed in the Supplementary Materials.

\section{Mouse models}

The mouse model of HBx protein-induced HCC was constructed based on Fah-deficient mice [13]. The $\mathrm{Fah}^{-/-}$mice were maintained with $7.5 \mu \mathrm{g} / \mathrm{mL}$ 2-(2-nitro-4-trifluoromethylbenzoyl)-1, 3-cyclohexanedione (NTBC). At the age of 5-7 weeks, mice were injected with 15- $\mu \mathrm{g}$ constructs containing Fah cDNA and the HBx mutants or WT by hydrodynamic injection via tail vein. After the injection, NTBC was removed from the drinking water. Mice that died within 30 days after the injection were excluded from the following analyses due to the failure in gene delivery. All mice were sacrificed on Day 150 after the infection. The livers and tumors were pathologically examined. Hematoxylin-eosin (H\&E) and immunohistochemistry $(\mathrm{IHC})$ are detailed in the Supplementary Materials. The antibodies used for IHC are detailed in Table S3.

Five-week-old Nod-SCID mice were purchased from Jihui Laboratory Animal Care Cooperation (Shanghai, China). Mice were subcutaneously injected with $1.5 \times 10^{6} \mathrm{HeL}$ a cells stably expressing wild-type or mutant HBx protein. Five weeks later, tumors were harvested. All animal studies were conducted under the animal welfare protocol approved by the ethics committee of Second Military Medical University.

\section{HBV capture sequencing}

Seven liver tissues and seven tumor tissues of the $S B$ mouse models were subjected to HBV-capture sequencing. Liver tissues were from the mice without tumors, including three WT-HBx mice, one M3-HBx mouse, and three Ct-HBx mice. Tumor tissues were from one WT-HBx mouse, three M3-HBx mice, and three Ct-HBx mice. DNA was extracted from tissues using Genomic DNA Mini Kit (Invitrogen, Carlsbad, CA). The DNA libraries were constructed using the Fast Library Prep Kit (iGeneTech, Beijing, China). The capture probes of HBV panel were synthesized by iGeneTech. The libraries were captured using the probes of HBV panel and TargetSeq one enrichment kits (iGeneTech). The HiSeqTM 2500 platform (Illumina, San Diego, CA) was applied for the sequencing. The breakpoints of HBV integration were detected using VERSE software [14]. The annotation for the integrated breakpoints was performed with ANNOVAR software [15]. Data of HBV-capture sequencing were uploaded to the Sequence Read Archive (SRA) database under Accession Number PRJNA743432.

\section{Cytokine assessment}

The cytokine levels in mice plasma were measured by multiplex immunoassay with ProcartaPlex Kit (ThermoFisher Scientific, MA). In toal, 11 cytokines were tested: interleukin-4 (IL-4), IL-5, IL-6, IL-1ß, IL-12, interferon-a (IFNa), IFNy, tumor necrosis factor-a (TNFa), granulocyte macrophage colony stimulating factor (GM-CSF), transforming growth factor $\beta$ (TGF $\beta$ ), and vascular endothelial growth factor (VEGF). 


\section{Microarray profiling analysis}

HeLa cells stably expressing WT-HBx and the four HBx mutants were subjected to CDNA microarray assay by using Human Transcriptome Array 2.0 (Affymetrix, Santa Clara, CA). Differently expressed genes between WT-HBx group and each of the mutant HBx groups were identified using the edgeR package [16]. The significantly expressed genes were subjected to gene set enrichment analysis (GSEA) using GSEA software (http://software.broadinstitute.org/gsea/). Based on the significantly enriched genes, proteinprotein interaction (PPI) networks were generated using STRING v10 software (https://string-db.org/) [17]. Three liver tissues from WT-HBx mice, three tumor tissues from M3-HBx mice, and three tumor tissues from $\mathrm{Ct}-\mathrm{HBx}$ mice were also subjected to cDNA microarray analysis. The samples were randomly selected from each mouse group. Agilent-074809 SurePrint G3 Mouse GE V2.0 microarray (Agilent Technologies, Santa Clara, CA) was applied for this analysis. Mouse genes were converted to human homologs using the HUGO Gene Nomenclature Committee database. The gene profiling data of the $S B$ mouse models and HeLa cells were uploaded to the Gene Expression Omnibus (GEO) database under Accession Numbers GSE179125 and GSE179126, respectively.

\section{Luciferase reporter assay}

Promoter region was defined as 2000 bp upstream of the transcription start site of a given gene based on the Ensembl database (GRCh38.p13, https://asia.ensembl.org/Homo_sapiens). The promoter fragments of $C D C 20, P A / 1$, and $P 21$ were synthesized and sequenced by Obio Technology (Shanghai, China). The correct sequences were inserted into the firefly luciferase reporter vector (pGL4.10-basic vector, Promega, Madison, WI) to evaluate the promoter activity. HepG2 and HeLa cells were co-transfected with the constructed reporter vectors and Renilla luciferase vectors (pRL-TK, Promega). Luciferase activities were tested using a dual-luciferase reporter assay system (Promega). Firefly luciferase activity was normalized to Renilla luciferase activity.

\section{Statistical analysis}

Differences in continuous variables were determined by Student's $t$-test. For non-normal data, the Wilcoxon sum rank test was used. The analyses of the data from in vitro and in vivo experiments were performed with GraphPad Prism version 5.0 (GraphPad Software, San Diego, CA). All statistical tests were two-sided. $P<0.05$ was considered statistically significant.

\section{Results}

\section{Effects of WT-HBx and the HBx mutants on hepatocarcinogenesis in SB mice}

Empty SB vector and those carrying WT-HBx and the HBx mutants were successfully delivered into 42 mice. None of the empty vector-injected mice $(n=9)$ developed tumors. Tumors and shrunken and cirrhotic livers were evident in M2-HBx-, M3-HBx-, and Ct-HBx-injected mice (shown in Fig 1). The incidence of tumors was $14.3 \%$ (1/7), $50 \%(2 / 4), 42.8 \%$ (3/7), 87.5\% (7/8), and $57.1 \%(4 / 7)$ in the $S B$ models whose hepatic genomic DNA was integrated with WT-HBx, M1-HBx, M2-HBx, M3-HBx, and Ct-HBx, respectively. 
Compared to WT-HBx-injected mice, all HBx mutants-injected mice displayed a trend toward higher tumor burden, especially the M3-HBx and Ct-HBx groups. No apparent pathological change was observed in the livers of the vector-injected mice. Inflammatory cell infiltration and cancer nests were observed in the HBx mutants-injected mice (shown in Fig 2A), which were similar to the histopathological changes of mouse hepatitis-induced HCC [18]. Histopathologic analyses revealed that inflammatory cell infiltration was more severe in livers injected with HBx mutants than in those with WT-HBx. Our IHC analysis confirmed that the tumors were positive for HBx protein as well as cytokeratin 18 (CK18) and a-fetoprotein (AFP), the classic biomarkers of HCC (shown in Fig 1).

\section{The insertion sites of HBx fragments}

The integration sites of WT-HBx and the HBx mutants in the $S B$ mice were examined via HBV-capture sequencing. In total, 1750 integrations were identified (Table S4). HBx fragments were mostly integrated in the intergenic and intron regions, with only $3.71 \%$ located in the exon regions. There were nine genes with the insertion sites detected in more than three samples (Table S5). The first three most frequently integrated genes were Fah (42.8\%), catenin alpha 3 (Ctnna3) (28.6\%), and fragile histidine triad gene (Fhit) (28.6\%). Gene encoding telomerase reverse transcriptase (TERT) was not an integration size. The nine frequently integrated genes have low ratios of the reads with $\mathrm{HBx}$ integration to the normal reads, ranging from $0.09 \%$ to $0.25 \%$. The difference in the levels of integration sites was not significant between liver tissues and tumor tissues (shown in Fig 2B). The number of integration sites was not significantly different among WT-HBx-, M3-HBx-, and Ct-HBx-injected $S B$ mice (shown in Fig 2C). These data suggest that HCCs developed in the $S B$ mice were induced by the HBx proteins, rather than insertional mutations.

\section{Serum levels of proinflammatory cytokine in WT-HBx and the HBx mutants SB mouse models}

The levels of Th1 cytokines (IFNy, TNFa, IL-1 $\beta$, and IL-12), Th2 cytokines (IL-4 and IL-5), IL-6, IFNa, TGF $\beta$, VEGF, and GM-CSF were detected in the sera of the $S B$ mice (shown in Fig 3 and Fig S3). Among the Th1 cytokines, IFNy and TNFa were positive in all groups (shown in Fig 3A, 3B). The level of IFNy was significantly higher in the M3-HBx-injected mice than in the controls. The positivity rate of IL-1 $\beta$ was significantly higher in M2-HBx- and M3-HBx-injected mice than in the empty-vector-injected mice (shown in Fig 3C). IL-5 and IL- 6 were measurable in all samples. The levels of IL-5 and IL-6 were significantly higher in M3-HBx mice than in the empty vector- and WT-HBx-injected mice (shown in Fig 3E, 3F). The levels of IFNy, TNFa, IL-5, and IL-6 were significantly higher in the mice with tumors than in the tumor-free mice (shown in Fig $3 \mathrm{G}$ ). The positive rate of IL-1 $\beta$ was also significantly higher in the mice with tumors (shown in Fig $3 \mathrm{H}$ ). IFNa was only positive in two M3-HBx-injected mice and one Ct-HBx-injected mouse (shown in Fig S3). IL-4 was only positive in one Ct-HBx-injected mouse. The HBx mutants did not upregulate the expression of TGF- $\beta$, VEGF, and GM-CSF (shown in Fig S3).

\section{Effects of the HBx mutations on malignant phenotypes of cancer cells}

To investigate whether the function of the $\mathrm{HBx}$ mutation was hepatocyte-specific, we evaluated the oncogenic effects of the HBx mutants in HepG2 and HeLa cells. The expression of WT-HBx and HBx 
mutants (M1-HBx, M2-HBx, M3-HBx, and Ct-HBx) in HepG2 and HeLa cells via the lentiviral infection was confirmed by Western blot (shown in Fig S4A). Ectopic expression of M1-HBx, M2-HBx, M3-HBx, and Ct$\mathrm{HBx}$ significantly increased cell proliferation and the $S$ phase proportion of HepG2 and HeLa, compared to WT-HBx (shown in Fig 4A, 4B). No significant difference in migration and invasion was observed between HepG2 cells expressing WT-HBx and those expressing HBx mutants; the same was true for HeLa cells (shown in Fig $4 C$ and shown in Fig S4B-D). The effects of HBx mutants on cell proliferation, cell cycle, and migration were repeated in HeLa cells (shown in Fig 4D-F). As M3-HBx and Ct-HBx displayed the strongest tumorigenic capability in the $S B$ mouse models, the effects of M3-HBx and Ct-HBx on the growth of cancer cells were further investigated using the xenograft of HeLa cells in nude mice (HepG2 is not tumorigenic in nude mice). It was found that the tumor weight was significantly higher in HeLa cells with M3-HBx than in those with WT-HBx (shown in Fig 4G). Thus, the HBx mutants, especially M3-HBx and $\mathrm{Ct}-\mathrm{HBx}$, promote cancer development, and this effect is not hepatocyte-specific.

\section{Gene expression profiles affected by the HBx mutants}

Based on cDNA microarray assay of HeLa cells, we identified the differentially expressed genes in each of the mutant HBx groups relative to WT-HBx group. The PPI network for each HBx mutant group was generated using STRING software. Six hub genes were selected because they had strong PPI function (the highest STRING score) in at least one PPI network (shown in Fig S5A). The six hubs are E3 ubiquitin protein ligase (also named MDM2), replication factor C subunit 3 (RFC3), polo-like kinase 1 (PLK1), cell division cycle 6 (CDC6), early growth response 1(EGR1), and PAl1. The effects of HBx mutants on the expression of these hub genes were also investigated with the microarray data of the $S B$ mouse models with the highest tumor burden, M3-HBx and Ct-HBx. WT-HBx mice served as control. The expression levels of PAl1 were significantly higher in M3-HBx mice (fold change $(\mathrm{FC})=16.93, P<0.001$ ) and Ct-HBx mice $(\mathrm{FC}=3.36, P=0.001)$ than in WT-HBx control (Table S6).

The differentially expressed genes identified in HeLa cells and those identified in M3-HBx- and Ct-HBXinjected mice vs. WT-HBx-injected mice were subjected to GSEA analysis. Four cancer-related gene sets [19-22] were identified in both microarray assays: BERENJENO_TRANSFORMED_BY_RHOA_UP, MARKEY_RB1_CHRONIC_LOF_UP, VERNELL_RETINOBLASTOMA_PATHWAY_UP, and CHIANG_LIVER_CANCER_SUBCLASS_PROLIFERATION_UP. The first three gene sets were significantly enriched by $\mathrm{M} 3-\mathrm{HBx}$ and $\mathrm{Ct}-\mathrm{HBx}$ in the cell and tissue microarray data. The remaining one was significantly enriched by M3-HBx in both cell and tissue microarray data. The four cancer-related gene sets included PAI1, P21, SKP2, and CDC20, respectively (shown in Fig 5A-D). PAl1 is the hub gene of the PPI network; P21 and CDC20 were also identified as functional molecules in the PPI network. P21 was significantly upregulated in both $\mathrm{M} 3-\mathrm{HBx}$-injected mice $(\mathrm{FC}=3.26, P=0.006)$ and $\mathrm{Ct}-\mathrm{HBx}$-injected mice $(\mathrm{FC}=1.77, P=0.009)$. CDC20 was significantly upregulated in $\mathrm{M} 3-\mathrm{HBx}$-injected mice $(\mathrm{FC}=2.64, P$ $=0.005)$. SKP2, a molecule previously identified to be involved in HBx-induced carcinogenesis [10], was not identified in the PPI analysis. M3-HBx- and Ct-HBx-injected mice had a trend toward a higher level of SKP2 (M3-HBx, FC = 1.66, $P=0.08$; Ct-HBx, FC = 1.67, $P=0.09$ ), compared to WT-HBx-injected control. We also identified four inflammation-related gene sets [23-27] in the $S B$ models. IL-6-related gene set, 
CROONQUIST_IL6_DEPRIVATION_DN, was significantly enriched in the M3-HBx- and Ct-HBx-injected mice (shown in Fig S5B, S5C). The IFN- $\gamma$ receptor 1-related gene set,

MATSUDA_NATURAL_KILLER_DIFFERENTIATION, and an inflammatory response-related gene set, NEMETH_INFLAMMATORY_RESPONSE_LPS_DN, were significantly enriched in the M3-HBx-injected mice (shown in Fig S5D, S5E). In total, 317 genes were involved in the four cancer-related gene sets, while 197 genes were involved in the four inflammation-related ones. Overall, 77 genes overlapped in the cancerrelated gene sets and the inflammation-related gene sets. In total, 72 genes were involved in the two IL-6related gene sets enriched in M3-HBx- and Ct-HBx-injected mice. Interestingly, of the 72 genes, 55 (76.3\%), including P21, SKP2, and CDC20, were also included in the cancer-related gene sets.

\section{Effects of the HBx mutants on the expression of functional molecules}

We then investigated the expression levels of the functional molecules identified in the above bioinformatics in HepG2 and HeLa cells stably expressing WT-HBx or HBx mutants. The transcription levels of MDM2, RCF3, PLK1,CDC6, and EGR1 were not significantly affected by the four HBx mutants (shown in Fig S6). Interestingly, the mRNA and protein levels of PAl1 and CDC20 were significantly upregulated by M3-HBx and Ct-HBx in HepG2 and HeLa cells (shown in Fig 6A-D). HBx combo mutations (M1-HBx, M2-HBx, and M3-HBx) significantly downregulated the mRNA level and protein level of p21 in HepG2 rather than in HeLa cells (shown in Fig 6B, 6D). However, Ct-HBx significantly upregulated the expression of p21 in both HepG2 and HeLa cells. At the protein level, Ct-HBx significantly upregulated SKP2 in HeLa and HepG2 cells. The HBx mutants showed consistent effects on the RNA and protein expression of PAI1, CDC20, and p21, respectively. However, our luciferase assays showed that M3-HBX and Ct-HBx had no direct effects on the promoter activities of the three molecules, compared with WT-HBx in HepG2 and HeLa cell lines (shown in Fig S7). We then investigated the expression of PAI1, CDC20, and p21, the newly identified molecules participating in HBx-induced carcinogenesis, in the tumor tissue of the $S B$ mice. The protein levels of CDC20 and PAI1 were significantly higher in the tumors from M3-HBx and $\mathrm{Ct}-\mathrm{HBX}$-injected mice, compared to their expression in the tumors from WT-HBx-injected mice (shown in Fig 7A, 7B). The protein level of p21 in the tumors was significantly lower in the M2-HBx group than in the WT-HBx group.

\section{Reversal of HBx mutations' effects by silencing functional molecules}

To figure out if the HBx mutations regulate the biological activity of HepG2 and HeLa cells via upregulating PAI1, CDC20, and p21, we knocked-down the three genes and then examined the effect of stably expressed WT-HBx and HBx mutants on the proliferation of HepG2 and HeLa cells. PAl1 silencing significantly neutralized the effects of M3-HBx and Ct-HBx on the proliferation and cell cycle of HepG2 and HeLa cells. CDC20 silencing significantly reversed the effects of M3-HBx on the proliferation and cell cycle in HeLa cells. P21 silencing reversed the Ct-HBx-induced increase in the proportion of S phase cells, rather than cell proliferation in HeLa and HepG2 cells (shown in Fig 8).

\section{Discussion}


In this study, we found that the HBx mutants-injected mice displayed a trend toward higher tumor burden, especially the M3-HBx- and Ct-HBx-injected mice, than the WT-HBx counterpart. This result is consistent with a previous study demonstrating that the HBx with A1762T/G1764A (K130M/V131I) mutation (the same as M1-HBx in our study) had a stronger tumorigenic effect than its WT counterpart in a $S B$ mouse model [28]. M2-HBx and M3-HBx contain two more HCC-risk mutations [7-9]. M3-HBx- and Ct-HBxinjected mice were more tumorigenic than M1-HBx-injected mice. As WT-HBx and the HBx mutants were integrated into the hepatic genome, HCC development can be caused by the integration site such as TERT $[29,30]$. We then applied HBV-capture sequencing to identify the integration sites. TERT was not the target. The introduced WT-HBx or mutant HBx genes express HBx protein, as detected by IHC (shown in Fig. 1). The tumors were confirmed to be HCC as CK-18 and AFP were positive [31]. Thus, HCC developed in the $S B$ mice are induced by the HBx proteins, rather than the insertional mutations. To further determine the effects of HBx mutants on the growth, we introduced WT-HBx and the HBx mutants into HepG2 and HeLa cells, respectively. Ectopic expression of HBx mutants significantly increased cell proliferation and the $S$ phase proportion. Thus, the HBx mutants are more carcinogenic than WT-HBx. Of those, M3-HBX and $\mathrm{Ct}-\mathrm{HBx}$ are the most potent ones.

Our histopathologic analyses revealed more inflammation events in livers of the mice injected with M2$\mathrm{HBx}, \mathrm{M} 3-\mathrm{HBx}$, and Ct-HBx than their corresponding WT-HBx counterparts and even M1-HBx; furthermore, signs of necrosis, fibrosis, and degenerative changes were also evident in liver sections (shown in Fig. 1). The serum levels of IFNy, IL-5, and IL-6 were significantly higher in M3-HBx-injected mice than in the empty vector- or WT-HBx-infected controls, while the positive rate of IL-1 $\beta$ was significantly higher in M2$\mathrm{HBx}$ - and M3-HBx-injected mice than in the vector-injected mice (shown in Fig. 3). IFNy has both protumor and antitumor activities, although it induces the Th1-recruiting, proinflammatory chemokines in the tumor microenvironment [32]. IL-5 contributes to the differentiation and survival of eosinophils in the tumor microenvironment, which facilitates tumor progression [32, 33]. IL-6 basically promotes cancer immune evasion and facilitates the development of HCC [34]. IL-1 $\beta$, a pro-inflammatory cytokine abundantly expressed in the tumor microenvironment, plays a major role in cancer invasiveness, progression, and metastases. Anti-inflammation with canakinumab targeting IL-1 $\beta$ significantly reduces cancer occurrence and mortality [35]. The higher circulating levels of IFN- $\gamma$, IL-5, and IL- 6 and higher positivity rate of IL-1 $\beta$ are quite consistent with the higher occurrence of $\mathrm{HCC}$ in M3-HBx-injected mice, indicating M3-HBXcaused inflammation is closely related to HCC development. The three inflammatory related gene sets were significantly enriched in M3-HBx-injected mice, while the IL-6-related gene set was significantly enriched in Ct-HBx-injected mice. We found that 77 genes overlapped in the cancer-related gene sets and the inflammation-related gene sets. Of the 72 genes in the IL-6-related gene sets, 55 (76.3\%), including CDC20, P21, and SKP2, were included in the cancer-related gene sets. These data indicate that the inflammatory (especially IL-6-related) signaling pathways are closely associated with the development of HBx mutants-caused HCC.

Ectopic expression of the HBx mutants significantly increased cell proliferation and the $S$ phase proportion of HepG2. These effects were exactly repeated in HeLa cells. Thus, the effects of the HBx mutants on cell biology are not hepatic-specific. HBV hepatotropism is mediated by specific receptor 
recognition in the liver [36]. After being introduced, the HBx mutants exhibit pro-carcinogenic effects in non-hepatic cells such as HeLa cells [37]. Due to the ease of cell culture and gene transfer, stable genome, and tumorigenicity in nude mice, HeLa is the most widely used human cancer cell line in elucidating the biochemical pathways of normal and diseased tissue in human cells [38]. Thus, HeLa cells were applied for cDNA microarray assay in this study. Using the PPI network, GSEA assay, and published data, we identified four key molecules (PAI1, CDC20, P21, and SKP2) in the four cancer-related gene sets that were quite consistent in GSEA between cell CDNA microarray assay in humans and tissue CDNA microarray assay in mice. The expression levels of SKP2 and p21 in HepG2 cells with M3-HBx are quite consistent with a previous study [10], indicating that our experiments are reliable. No study reports the effect of Ct$\mathrm{HBx}$ on p21 expression. Here, we found that the expression level of p21 was higher in Ct-HBx-transfected HepG2 and HeLa cells, possibly because the 3'-terminal of HBx inhibits the expression of p21 in cancer cells. The effects of the HBx mutants on CDC20 expression are also not reported. CDC20 is an activator of the anaphase-promoting complex/cyclosome. Recent studies have demonstrated that HBx induces mitotic checkpoint dysfunction via targeting the human serine/threonine kinase BubR1, while HBx binding to BubR1 attenuates the association between BubR1 and CDC20 [37, 39]. Ct-HBx and HBx mutations at the Kunitz domains may fail to bind BubR1, thus facilitating the binding of hBubR1 to CDC20. However, this could not explain why Ct-HBx and M3-HBx upregulate the expression of CDC20 in HepG2 and HeLa cells and in the liver of corresponding SB mice. The reason that the effect of M3-HBx on the growth of HeLa cells is attenuated by silencing CDC20 remains to be elucidated. The mechanisms by which the HBx mutants upregulate the expression of the four key molecules remain unknown. Our reporter assays indicated that the HBx mutants do not trans-activate the promoter activities of PA/1, $C D C 20$, and $p 21$. It is possible that the HBx mutants trans-activate their enhancers or stabilize their mRNAs via epigenetic modifications.

For the first time, we found that PAl1 whose expression is significantly upregulated by Ct-HBx or M3-HBx in cell lines and in the $S B$ mice functions as a hub. Most importantly, silencing PAl1 attenuates the effect of M3-HBx or Ct-HBx on the growth of HepG2 cells and significantly decreases the growth of HeLa cells with Ct-HBx. Thus, PAl1 is an important player in HBx mutants-induced carcinogenesis. PAl1 (also known as SERPINE1) is a multifaceted proteolytic factor that not only has anti-fibrinolytic and anti-plasminogen activation activities but also plays a pro-tumorigenic role via its pro-angiogenic and anti-apoptotic activities [40]. In the tumor microenvironment, PAl1 expression is upregulated in tumor-associated macrophages after being stimulated with cancer-associated fibroblasts-generating CXCL12 and promotes the malignant behavior of the HCC cells by mediating epithelial-mesenchymal transition [41]. High levels of PAl1 and its genetic predisposition have been consistently reported to predict poor prognosis in several types of human cancers including HBV-HCC [41-44]. Thus, the HBx mutants upregulate the expression of PAl1, facilitating the generation of the cancer-promoting microenvironment in affected livers. Thus, PAI1 is a promising candidate as a predictive and/or prognostic biomarker and a therapeutic target in HBV-HCC.

Our study has limitations. First, we did not elucidate the mechanism by which the HBx mutants upregulate the expression of PAI1, CDC20, and p21, although we exclude the possibility that the HBx 
mutants trans-activate their promoters. Second, the effect of the 3'-terminal of HBx on the expression of p21 was not investigated. Third, the mechanisms by which M2-HBx, M3-HBx, and Ct-HBx induced cancerpromoting inflammation remain to be investigated.

\section{Conclusions}

Conclusively, the HBx combo mutation C1653T + T1674G + A1762T/G1764A promotes carcinogenesis via upregulating the expression of PAl1 and $\mathrm{CDC} 20$ and inducing cancer-promoting inflammation. The oncogenic effect of Ct-HBx also depends on the upregulation of PAl1. Thus, PAl1 should be an important predictive and prognostic biomarker and promising therapeutic target in HBV-HCC.

\section{Abbreviations}

HBV, hepatitis B virus; HCC, hepatocellular carcinoma; HBx, hepatitis B virus X; SB, Sleeping Beauty; PAl1, plasminogen activator inhibitor-1; CDC20, cell division cycle 20; M1, A1762T/G1764A; M2, T1674G + T1753C + A1762T/G1764A; M3, C1653T + T1674G + A1762T/G1764A; Ct-HBx, carboxylic acid-terminal truncated HBx; WT, wild-type; Fah, fumaryl acetoacetate hydrolase; NTBC, 2-(2-nitro-4trifluoromethylbenzoyl)-1,3-cyclohexanedione; IHC, Immunohistochemistry; H\&E, hematoxylin-eosin; GEO, Gene Expression Omnibus; IL-4, interleukin-4; IFN-a, interferon- $a$; TNF- $a$, tumor necrosis factor- $a$; GM-CSF, granulocyte macrophage colony stimulating factor; TGF $\beta$, transforming growth factor $\beta$; VEGF, vascular endothelial growth factor; DMEM, Dulbecco's modified Eagle's medium; STR, short tandem repeat; siRNAs, Small interfering RNAs; P21(CDKN1A), cyclin dependent kinase inhibitor 1A; qRT-PCR, real-time quantitative reverse transcription PCR; CCK8, Cell Counting Kit-8; GSEA, gene set enrichment analysis; PPI, protein-protein interaction; GAPDH, Glyceraldehyde- 3-phosphate dehydrogenase; CK18, cytokeratin 18; Afp, alpha-fetoprotein; Ctnna3, catenin alpha 3; Fhit, fragile histidine triad gene; MDM2, E3 ubiquitin protein ligase; RCF3, replication factor C subunit 3; PLK1, polo like kinase 1; CDC6, cell division cycle 6; EGR1, early growth response 1; SKP2, S-phase kinase associated protein 2.

\section{Declarations}

\section{Ethics approval and consent to participate}

All animal studies were conducted under the animal welfare protocol approved by the ethics committee of Second Military Medical University.

\section{Consent for publication}

All authors agree to submit the article for publication.

\section{Availability of data and materials}


Data of HBV-capture sequencing were uploaded to the Sequence Read Archive (SRA) database under Accession Number PRJNA743432. The gene profiling data of the SB mouse models and HeLa cells were uploaded to the Gene Expression Omnibus (GEO) database under Accession Numbers GSE179125 and GSE179126, respectively.

\section{Competing interests}

The authors declare that they have no competing interests.

\section{Funding}

This work was supported by grant 2015CB554006 from the National Key Basic Research Program of China (GC); grants 91529305 (GC), 81520108021 (GC), 81673250 (GC), 81521091 (GC), 82003538 (WL), and 81502882 (XC) from the National Natural Science Foundation of China, grant 20 YF1458800 (WL) from the Shanghai Yangfan Program, and grants GWV-10.1-XK17 from the "3-year public health promotion" program of Shanghai Municipal Health Commission (GC).

\section{Author Contributions}

Cao GW conceived and supervised the study. Pu R, Zhou XY, Hou XM, Cai SL, Chen LP, Wu JF, and Yang F carried out the experiments. Chen $X$ did the bioinformatics analysis. Liu WB and Zhou XY conducted the statistical analyses and drafted the manuscript. Cao GW interpreted the data, discussed the results, and extensively revised the manuscript. Wang X provided the Sleeping Beauty mouse model. Tan XJ and Yin $\mathrm{JH}$ guided on the experiments. All authors read and approved the final manuscript.

\section{Acknowledgements}

Not applicable.

\section{References}

1. Sung H, Ferlay J, Siegel RL, Laversanne M, Soerjomataram I, Jemal A, et al. Global Cancer Statistics 2020: GLOBOCAN Estimates of Incidence and Mortality Worldwide for 36 Cancers in 185 Countries. CA Cancer J Clin 2021;71:209-249.

2. Sagnelli E, Macera M, Russo A, Coppola N, Sagnelli C. Epidemiological and etiological variations in hepatocellular carcinoma. Infection 2020;48:7-17.

3. de Martel C, Maucort-Boulch D, Plummer M, Franceschi S. World-wide relative contribution of hepatitis B and C viruses in hepatocellular carcinoma. Hepatology 2015;62:1190-200.

4. Yang F, Ma L, Yang Y, Liu W, Zhao J, Chen X, et al. Contribution of Hepatitis B Virus Infection to the Aggressiveness of Primary Liver Cancer: A Clinical Epidemiological Study in Eastern China. Front Oncol 2019;9:370. 
5. Yin J, Chen X, Li N, Han X, Liu W, Pu R, et al. Compartmentalized evolution of hepatitis B virus contributes differently to the prognosis of hepatocellular carcinoma. Carcinogenesis 2021;42:461470.

6. Chauhan R, Michalak TI. Earliest hepatitis B virus-hepatocyte genome integration: sites, mechanism, and significance in carcinogenesis. Hepatoma Res 2021;7:20.

7. Yin J, Xie J, Liu S, Zhang H, Han L, Lu W, et al. Association between the various mutations in viral core promoter region to different stages of hepatitis $B$, ranging of asymptomatic carrier state to hepatocellular carcinoma. Am J Gastroenterol 2011;106:81-92.

8. Hayashi S, Khan A, Simons BC, Homan C, Matsui T, Ogawa K, et al. An association between core mutations in hepatitis B virus genotype F1b and hepatocellular carcinoma in Alaskan native people. Hepatology 2019;69:19-33.

9. Yin J, Wang J, Pu R, Xin H, Li Z, Han X, et al. Hepatitis B virus combo mutations improve the prediction and active prophylaxis of hepatocellular carcinoma: a clinic-based cohort study. Cancer Prev Res (Phila) 2015;8:978-988.

10. Huang Y, Tong S, Tai AW, Hussain M, Lok AS. Hepatitis B virus core promoter mutations contribute to hepatocarcinogenesis by deregulating SKP2 and its target, p21. Gastroenterology 2011;141:14121421, 1421.e1-5.

11. Yin J, Li N, Han Y, Xue J, Deng Y, Shi J, et al. Effect of antiviral treatment with nucleotide/nucleoside analogs on postoperative prognosis of hepatitis B virus-related hepatocellular carcinoma: a twostage longitudinal clinical study. J Clin Oncol 2013;31:3647-3655.

12. Zhang Y, Yan Q, Gong L, Xu H, Liu B, Fang X, et al. C-terminal truncated HBx initiates hepatocarcinogenesis by downregulating TXNIP and reprogramming glucose metabolism. Oncogene 2021;40:1147-1161.

13. Wangensteen KJ, Wilber A, Keng VW, He Z, Matise I, Wangensteen L, et al. A facile method for somatic, lifelong manipulation of multiple genes in the mouse liver. Hepatology 2008;47:1714-1724.

14. Wang Q, Jia P, Zhao Z. VERSE: a novel approach to detect virus integration in host genomes through reference genome customization. Genome Med 2015;7:2.

15. Wang K, Li M, Hakonarson $\mathrm{H}$. ANNOVAR: functional annotation of genetic variants from highthroughput sequencing data. Nucleic Acids Res 2010;38:e164.

16. McCarthy DJ, Chen Y, Smyth GK. Differential expression analysis of multifactor RNA-Seq experiments with respect to biological variation. Nucleic Acids Res 2012;40:4288-4297.

17. Szklarczyk D, Franceschini A, Wyder S, Forslund K, Heller D, Huerta-Cepas J, et al. STRING v10: protein-protein interaction networks, integrated over the tree of life. Nucleic Acids Res. 2015 Jan;43(Database issue):D447-52.

18. Thoolen B, Maronpot RR, Harada T, Nyska A, Rousseaux C, Nolte T, et al. Proliferative and nonproliferative lesions of the rat and mouse hepatobiliary system. Toxicol Pathol 2010;38:5S-81S.

19. Berenjeno IM, Núñez F, Bustelo XR. Transcriptomal profiling of the cellular transformation induced by Rho subfamily GTPases. Oncogene 2007;26:4295-4305. 
20. Markey MP, Bergseid J, Bosco EE, Stengel K, Xu H, Mayhew CN, et al. Loss of the retinoblastoma tumor suppressor: differential action on transcriptional programs related to cell cycle control and immune function. Oncogene 2007;26:6307-6318.

21. Vernell R, Helin K, Müller H. Identification of target genes of the p16INK4A-pRB-E2F pathway. J Biol Chem 2003;278:46124-46137.

22. Chiang DY, Villanueva A, Hoshida Y, Peix J, Newell P, Minguez B, et al. Focal gains of VEGFA and molecular classification of hepatocellular carcinoma. Cancer Res 2008;68:6779-6788.

23. Croonquist PA, Linden MA, Zhao F, Van Ness BG. Gene profiling of a myeloma cell line reveals similarities and unique signatures among IL-6 response, N-ras-activating mutations, and coculture with bone marrow stromal cells. Blood 2003;102:2581-2592.

24. Matsuda JL, Zhang Q, Ndonye R, Richardson SK, Howell AR, Gapin L. T-bet concomitantly controls migration, survival, and effector functions during the development of Valpha14i NKT cells. Blood 2006;107:2797-2805.

25. Németh ZH, Leibovich SJ, Deitch EA, Vizi ES, Szabó C, Hasko G. cDNA microarray analysis reveals a nuclear factor-kappaB-independent regulation of macrophage function by adenosine. J Pharmacol Exp Ther 2003;306:1042-1049.

26. Chiu AP, Tschida BR, Sham TT, Lo LH, Moriarity BS, Li XX, et al. HBX-K130M/V131I promotes liver cancer in transgenic mice via AKT/FOX01 signaling pathway and arachidonic acid metabolism. Mol Cancer Res 2019;17:1582-1593.

27. Sze KM, Ho DW, Chiu YT, Tsui YM, Chan LK, Lee JM, et al. Hepatitis B virus-telomerase reverse transcriptase promoter integration harnesses host ELF4, resulting in telomerase reverse transcriptase gene transcription in hepatocellular carcinoma. Hepatology 2021;73:23-40.

28. Ma ZX, Yang CM, Li MG, Tu H. Telomerase reverse transcriptase promoter mutations in hepatocellular carcinogenesis. Hepatoma Res 2019;5:8.

29. Ismail SA, El Saadany Sh, Ziada DH, Zakaria SS, Mayah WW, Elashry H, et al. Cytokeratin-18 in Diagnosis of HCC in Patients with Liver Cirrhosis. Asian Pac J Cancer Prev 2017;18:1105-1111.

30. Gocher AM, Workman CJ, Vignali DAA. Interferon-y: teammate or opponent in the tumour microenvironment? Nat Rev Immunol 2021. doi: 10.1038/s41577-021-00566-3.

31. Quail DF, Olson OC, Bhardwaj P, Walsh LA, Akkari L, Quick ML, et al. Obesity alters the lung myeloid cell landscape to enhance breast cancer metastasis through IL5 and GM-CSF. Nat Cell Biol 2017;19:974-987.

32. Zaynagetdinov R, Sherrill TP, Gleaves LA, McLoed AG, Saxon JA, Habermann AC, et al. Interleukin-5 facilitates lung metastasis by modulating the immune microenvironment. Cancer Res 2015;75:16241634.

33. Ridker PM, MacFadyen JG, Thuren T, Everett BM, Libby P, Glynn RJ, et al. Effect of interleukin-1 $\beta$ inhibition with canakinumab on incident lung cancer in patients with atherosclerosis: exploratory results from a randomised, double-blind, placebo-controlled trial. Lancet 2017;390:1833-1842. 
34. Schieck A, Schulze A, Gähler C, Müller T, Haberkorn U, Alexandrov A, et al. Hepatitis B virus hepatotropism is mediated by specific receptor recognition in the liver and not restricted to susceptible hosts. Hepatology 2013;58:43-53.

35. Chae S, Ji JH, Kwon SH, Lee HS, Lim JM, Kang D, et al. HBxAPa/Rsf-1-mediated HBx-hBubR1 interactions regulate the mitotic spindle checkpoint and chromosome instability. Carcinogenesis 2013;34:1680-1688.

36. Masters JR. HeLa cells 50 years on: the good, the bad and the ugly. Nat Rev Cancer 2002;2:315-319.

37. Kim S, Park SY, Yong H, Famulski JK, Chae S, Lee JH, et al. HBV X protein targets hBubR1, which induces dysregulation of the mitotic checkpoint. Oncogene 2008;27:3457-3464.

38. Kubala MH, Punj V, Placencio-Hickok VR, Fang H, Fernandez GE, Sposto R, et al. Plasminogen activator inhibitor-1 promotes the recruitment and polarization of macrophages in cancer. Cell Rep 2018;25:2177-2191.e7.

39. Chen S, Morine Y, Tokuda K, Yamada S, Saito Y, Nishi M, et al. Cancer-associated fibroblast-induced M2-polarized macrophages promote hepatocellular carcinoma progression via the plasminogen activator inhibitor-1 pathway. Int J Oncol 2021;59:59.

40. Divella R, Daniele A, Abbate I, Savino E, Casamassima P, Sciortino G, et al. Circulating levels of PAI-1 and SERPINE1 4G/4G Polymorphism are predictive of poor prognosis in HCC patients undergoing TACE. TransI Oncol 2015;8:273-278.

41. Kubala MH, DeClerck YA. The plasminogen activator inhibitor-1 paradox in cancer: a mechanistic understanding. Cancer Metastasis Rev 2019;38:483-492.

42. Divella R, Mazzocca A, Gadaleta C, Simone G, Paradiso A, Quaranta M, et al. Influence of plasminogen activator inhibitor-1 (SERPINE1) 4G/5G polymorphism on circulating SERPINE-1 antigen expression in HCC associated with viral infection. Cancer Genomics Proteomics 2012;9:193198.

\section{Figures}




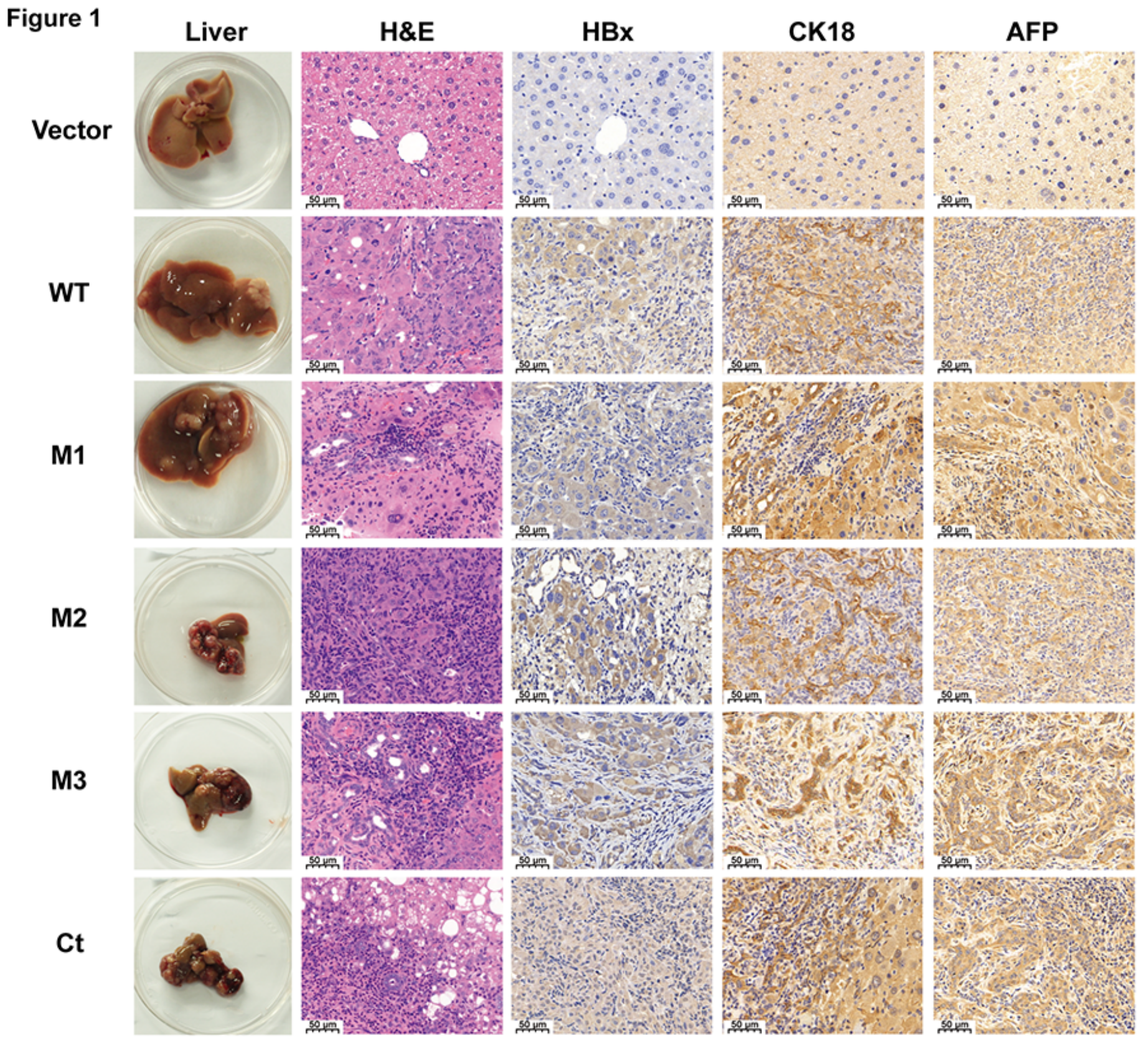

\section{Figure 1}

Representative features of H\&E and IHC stainings of the liver and tumors from the Sleeping Beauty (SB) mouse models. The first column shows representative gross features indicating the tumors in the livers. The second column shows representative H\&E stainings indicating the histological features of the livers. The third to fifth columns show representative IHC stainings for HBx, CK-18, and AFP. Vector, the mice injected with the empty vector; $\mathrm{WT}, \mathrm{M} 1, \mathrm{M} 2, \mathrm{M} 3$, and $\mathrm{Ct}$, the mice injected with vectors carrying WT-HBx, $\mathrm{M} 1-\mathrm{HBx}, \mathrm{M} 2-\mathrm{HBx}, \mathrm{M} 3-\mathrm{HBx}$, and Ct-HBx, respectively. 
Figure 2
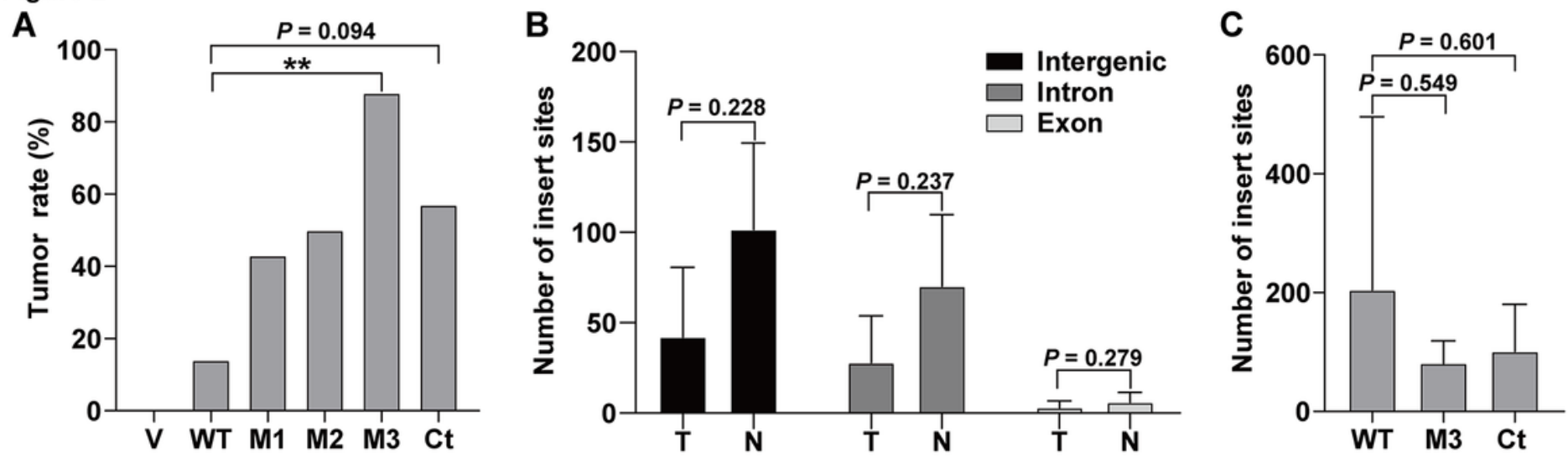

Figure 2

Tumor incidence and HBx insertion sites of the SB mouse models. (A) Incidence of tumors in the mouse models. V, the mouse models injected with the empty vector, $n=7 ; W T$, the mouse models injected with WT$\mathrm{HBx}, \mathrm{n}=7 ; \mathrm{M} 1$, the mouse models injected with $M 1-H B x, n=4 ; M 2$, the mouse models injected with M2-HBx, $\mathrm{n}=7 ; \mathrm{M} 3$, the mouse models injected with $\mathrm{M3}-\mathrm{HBx}, \mathrm{n}=8 ; \mathrm{Ct}$, the mouse models injected with $\mathrm{Ct}-\mathrm{HBx}, \mathrm{n}=7$.

(B) The number of $\mathrm{HBx}$ insertion sites in different functional regions and different tissue types. T, tumors from the mice with tumor nodules; $N$, liver tissues from the tumor-free mice. (C) The total number of HBx insertion sites between two of the three groups. ${ }^{*} \mathrm{P}<0.05$. 
Figure 3

A

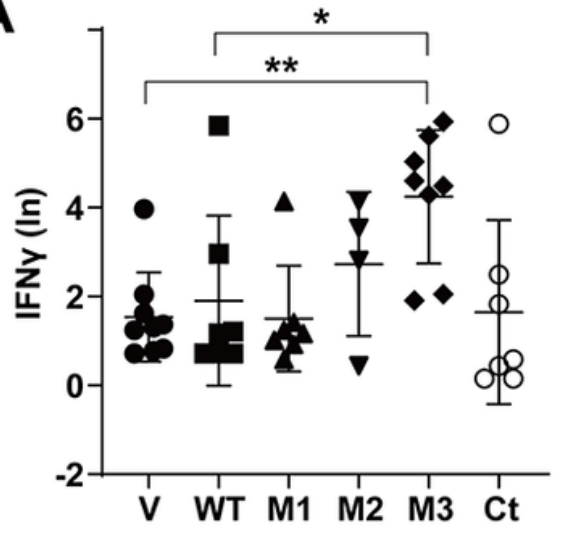

D

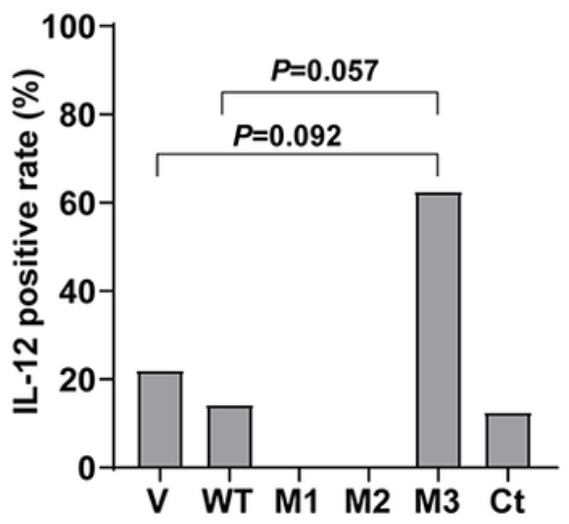

G

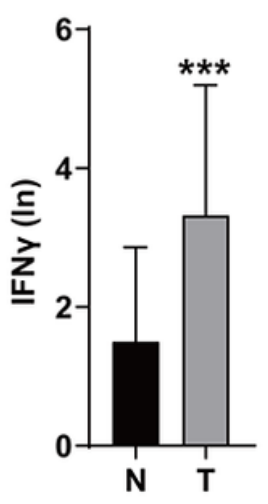

B

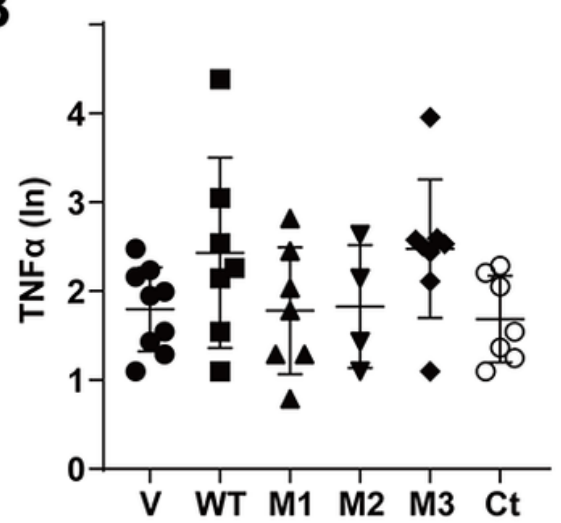

E

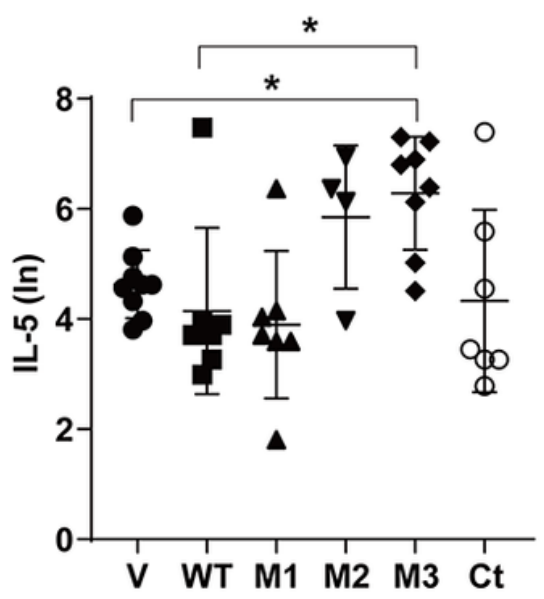

C

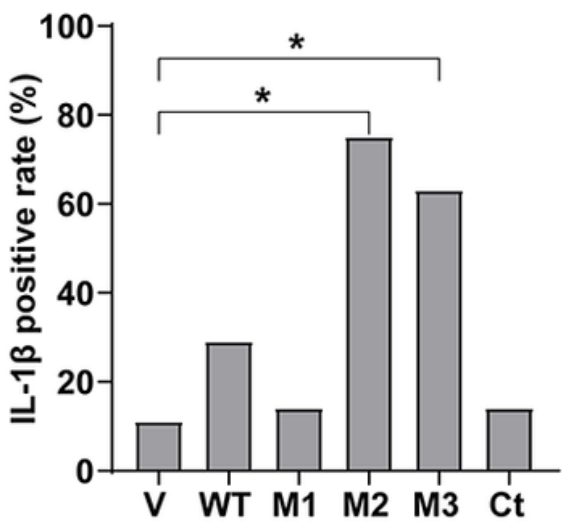

F

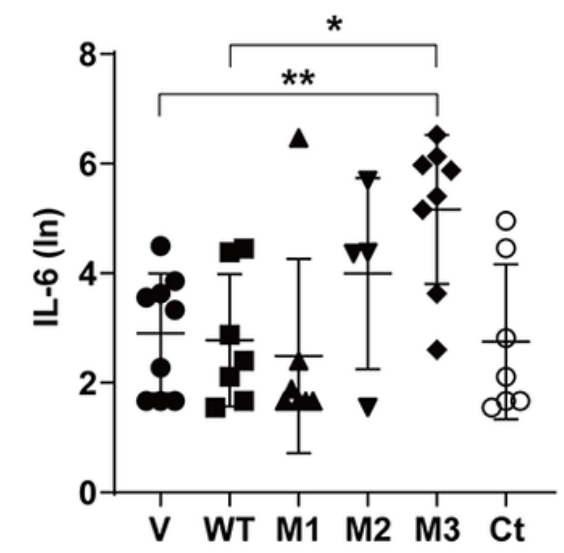

H
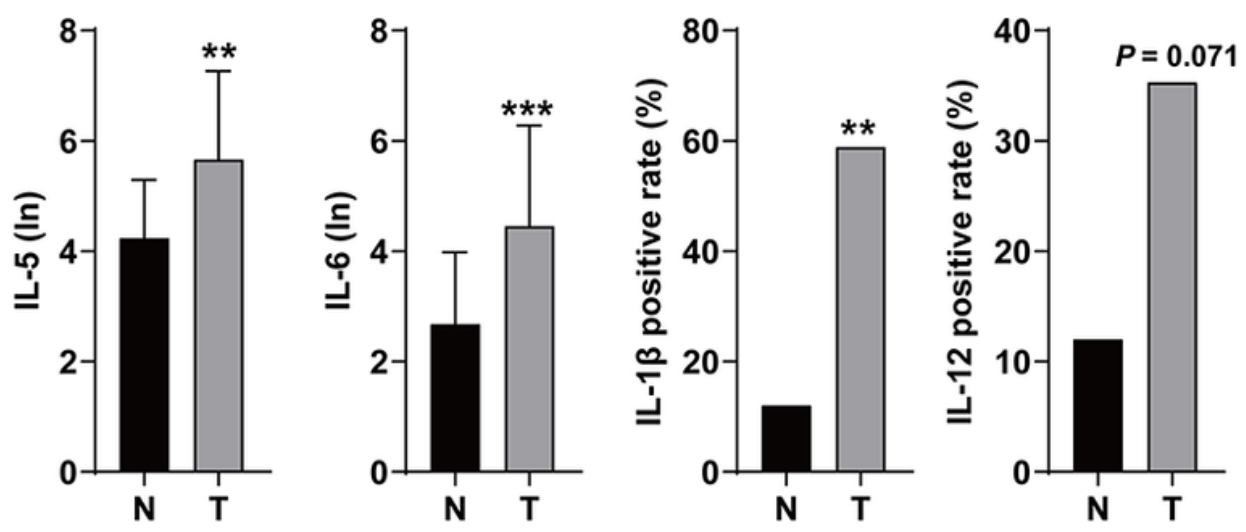

Figure 3

The serum levels and the positive rates of cytokines in the SB mouse models. (A) the serum level of IFN-y; (B) the serum level of TNF- $\alpha$; (C) the positive rate of IL-1 $\beta$; (D) the positive rate of IL-12; (E) the serum level of IL-5; (F) the serum level of IL- 6 ; (G) the serum levels of IFN-y, TNF-a, IL-5, and IL- 6 in the mouse models with or without tumor; and $(\mathrm{H})$ the positive rates of IL-1 $\beta$ and IL-12 in the mouse models with or without tumor. $\mathrm{N}$, the tumor-free mice; $\mathrm{T}$, the mice with tumors. ${ }^{*} \mathrm{P}<0.05 ; * \star \mathrm{P}<0.01 ; * \star \star \mathrm{P}<0.001$. 
Figure 4

A

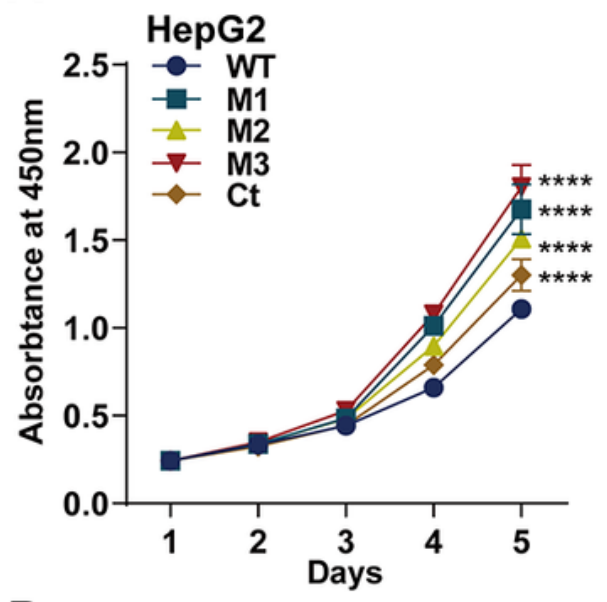

D

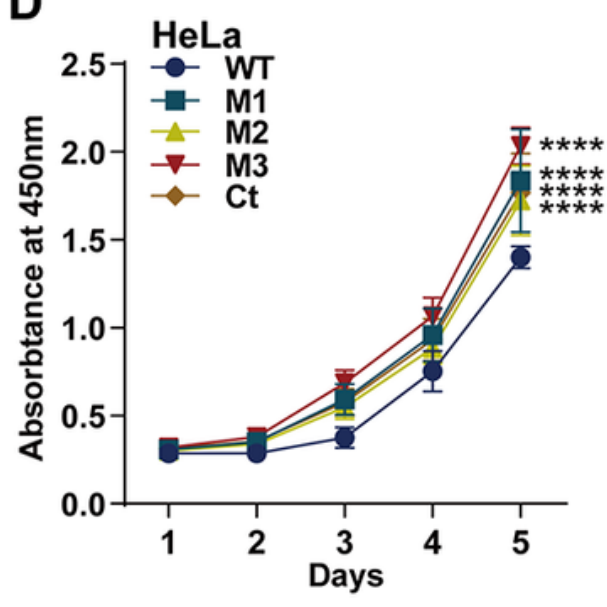

B

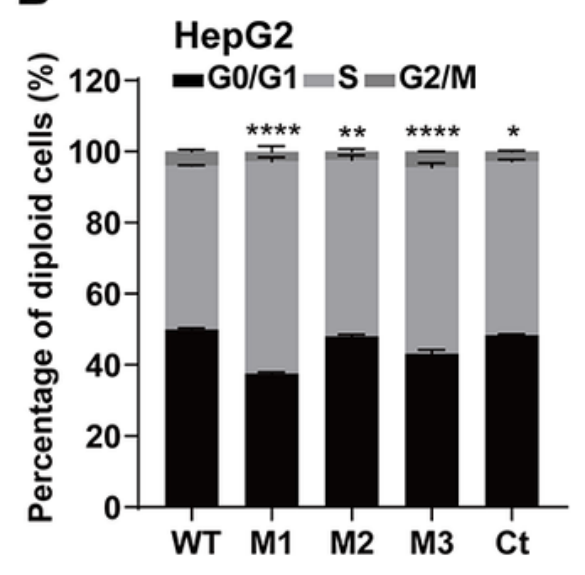

E

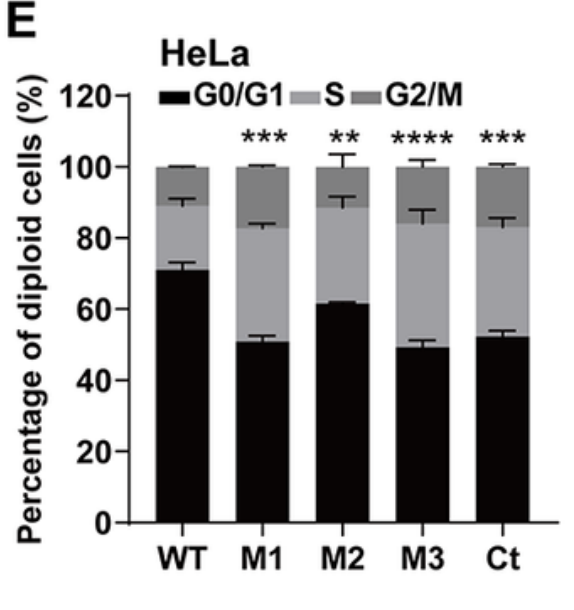

C

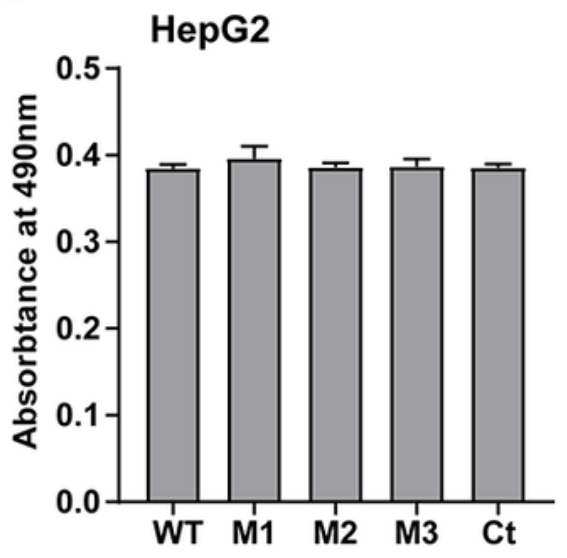

$\mathbf{F}$

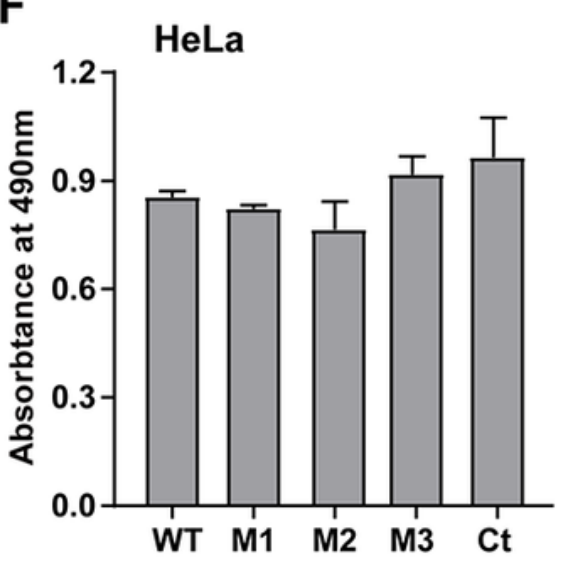

G
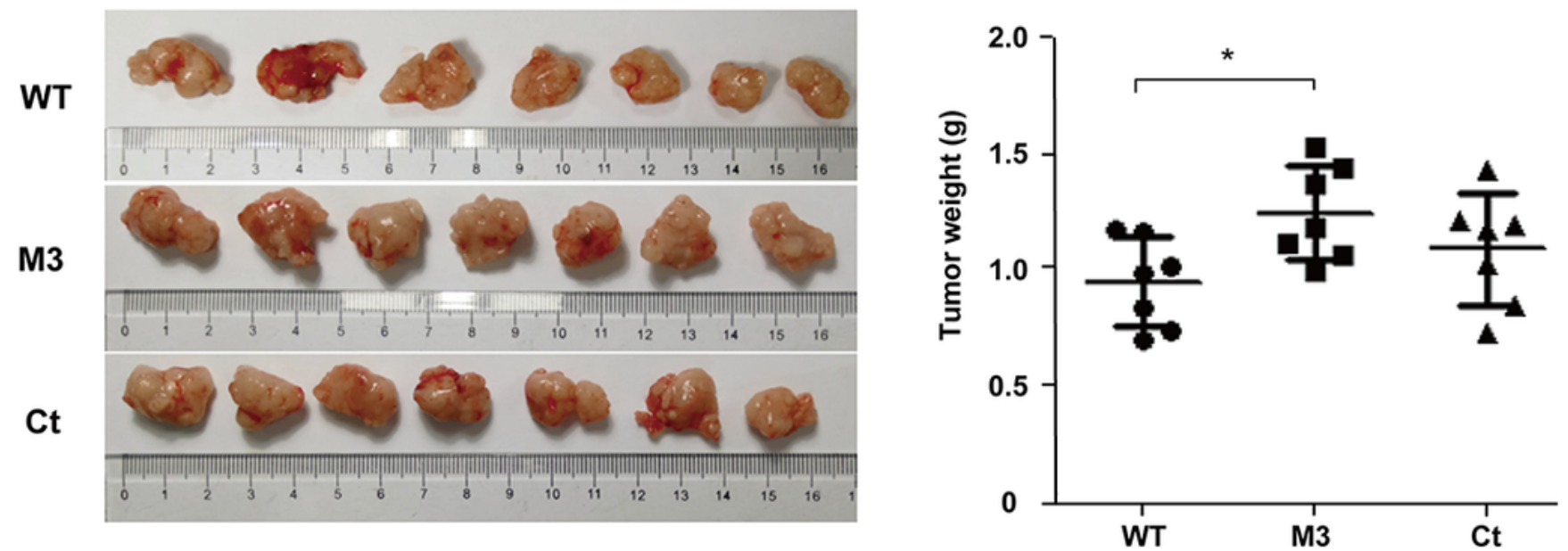

Figure 4

Effects of HBx mutations on malignant phenotypes of cancer cells. (A) Ectopic expression of the HBx mutants significantly increased the proliferation of HepG2 cells, compared to WT-HBX. (B) Ectopic expression of the HBx mutants increased the rate of cells in the S phase of HepG2 cells, compared to WTHBx. (C) Ectopic expression of the HBx mutants had no significant effect on the migration of HepG2 cells, compared to WT-HBx. (D) Ectopic expression of the HBx mutants significantly increased the proliferation 
of HeLa cells, compared to WT-HBx. (E) Ectopic expression of the HBx mutants increased the rate of cells in the S phase of HeLa cells, compared to WT-HBx. (F) Ectopic expression of the HBx mutants had no significant effect on the migration of HeLa cells, compared to WT-HBx. (G) Tumor weights among different groups. ${ }^{*} \mathrm{P}<0.05 ; * \star \mathrm{P}<0.01 ; * \star * \mathrm{P}<0.001 ; * \star \star * \mathrm{P}<0.0001$.

Figure 5

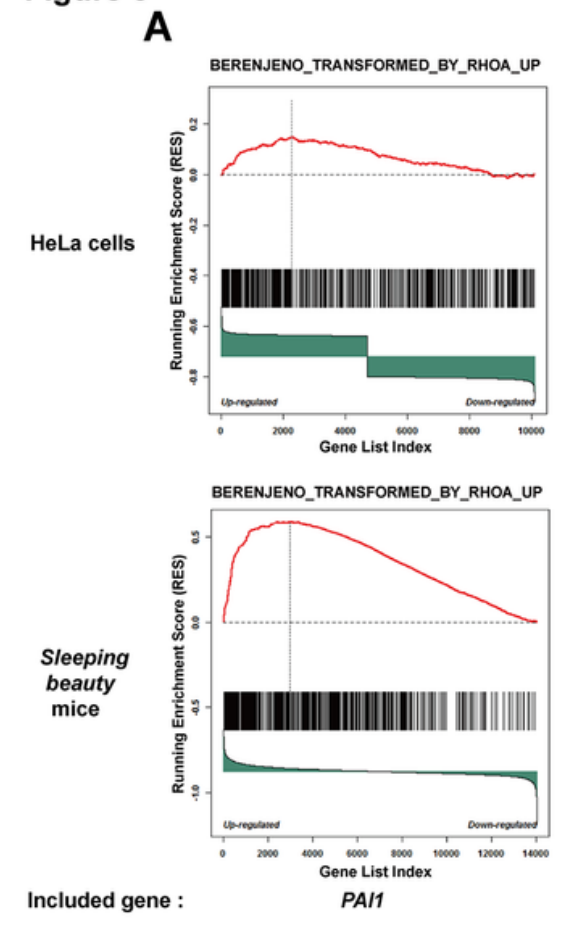

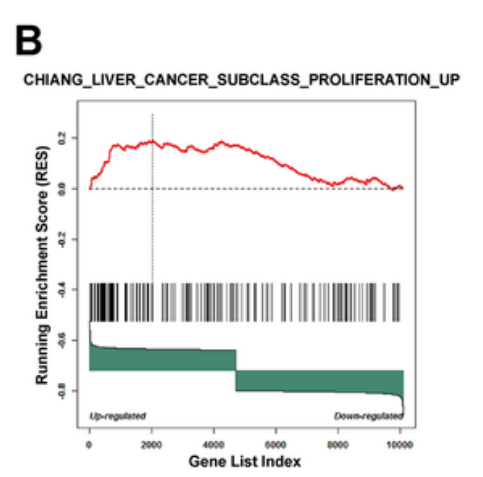
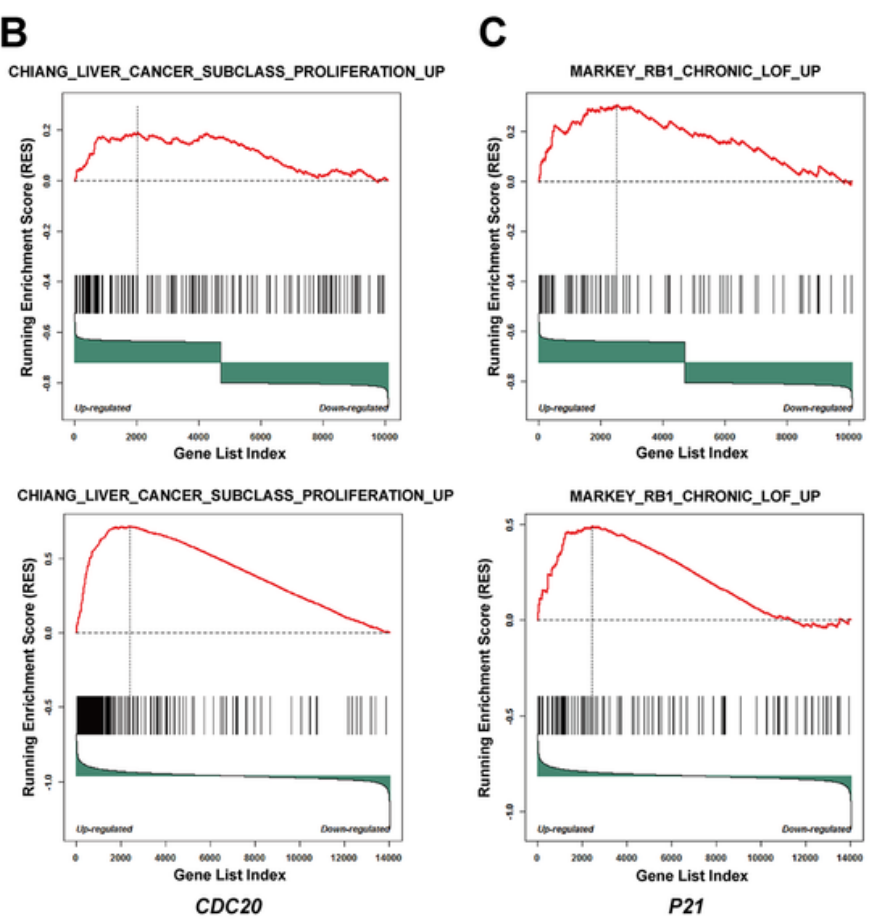
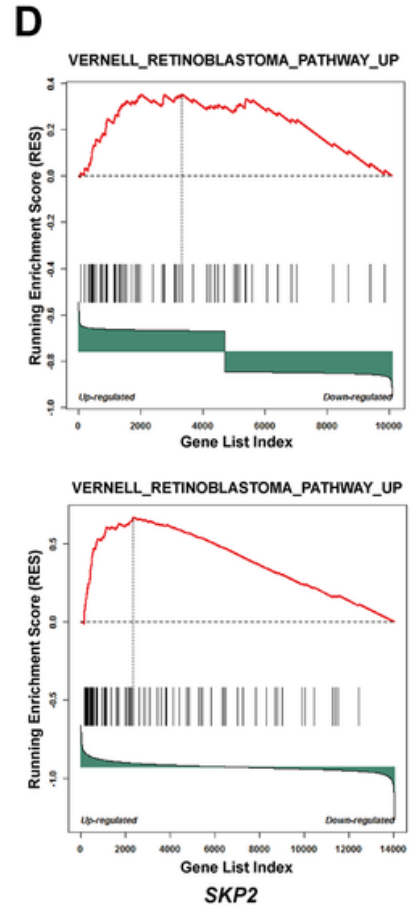

Figure 5

The four cancer-related gene sets identified both in human cell cDNA microarrays and in mouse tissue cDNA microarrays. (A) BERENJENO_TRANSFORMED_BY_RHOA_UP gene set including PAI1; (B) CHIANG_LIVER_CANCER_SUBCLASS_PROLIFERATION_UP gene set including CDC20; (C) MARKEY_RB1_CHRONIC_LOF_UP gene set including P21; and (D) VERNELL_RETINOBLASTOMA_PATHWAY_UP gene set including SKP2. 
Figure 6

A
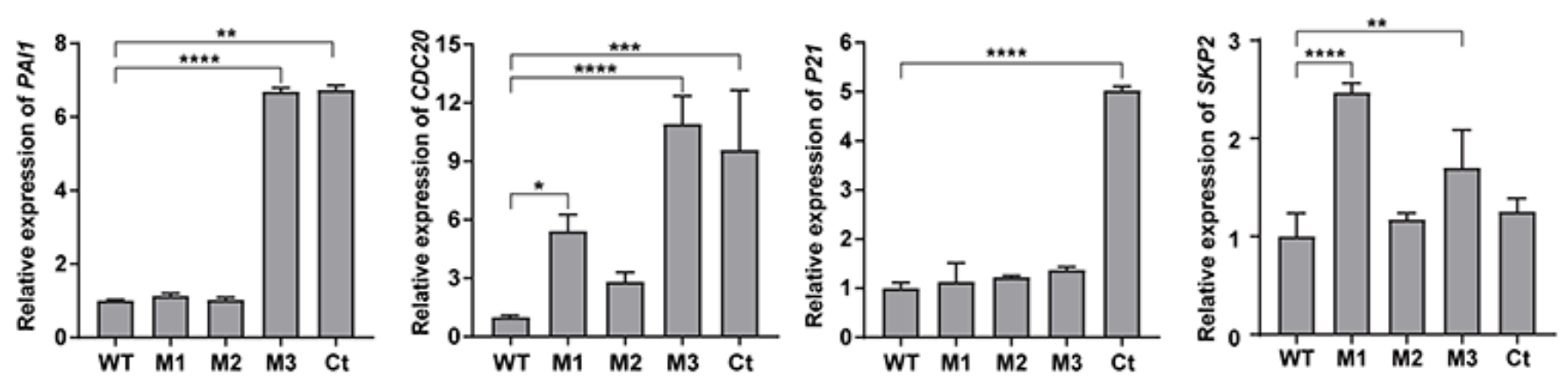

B
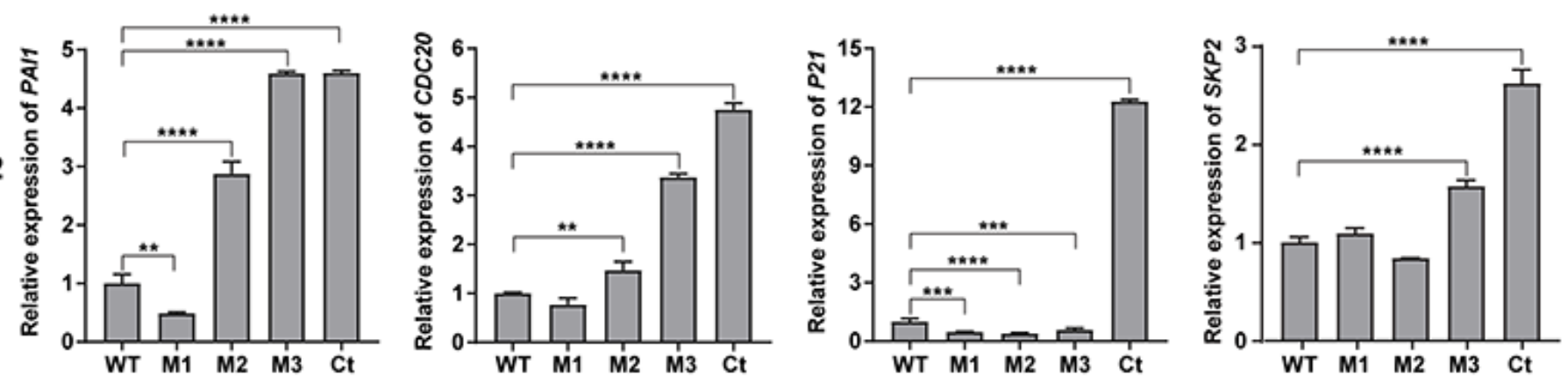

C

HeLa

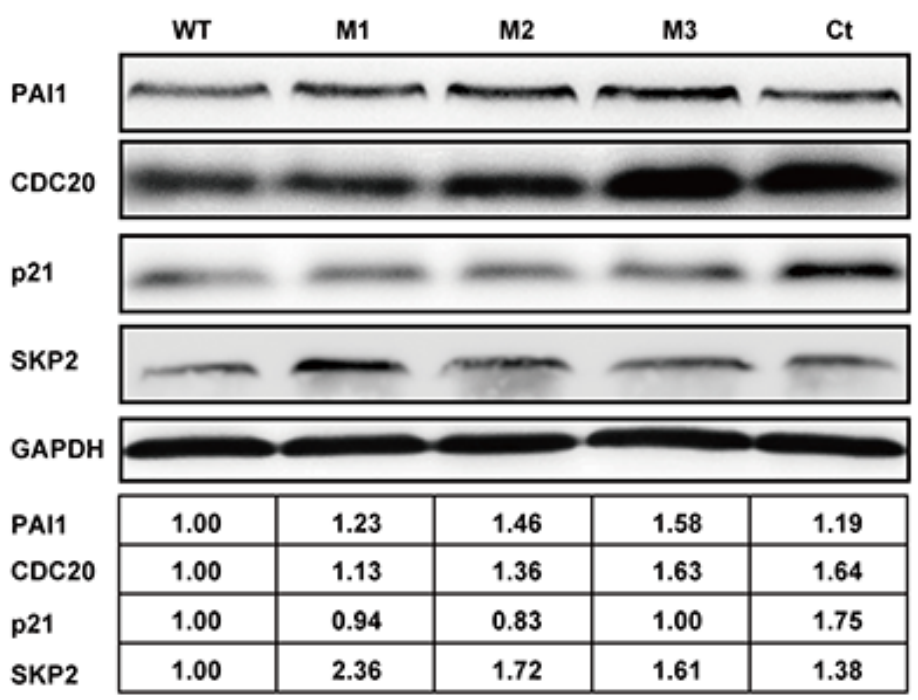

D

\section{HepG2}

\begin{tabular}{|c|c|c|c|c|c|}
\hline & WT & M1 & M2 & M3 & $\mathrm{Ct}$ \\
\hline \multicolumn{6}{|l|}{ PAl1 } \\
\hline \multicolumn{6}{|l|}{$\mathrm{CDC}_{20}$} \\
\hline \multicolumn{6}{|l|}{ p21 } \\
\hline \multicolumn{6}{|l|}{ SKP2 } \\
\hline \multicolumn{6}{|l|}{ GAPDH } \\
\hline PAl1 & 1.00 & 1.19 & 1.13 & 1.91 & 2.14 \\
\hline CDC20 & 1.00 & 1.11 & 1.38 & 1.42 & 2.13 \\
\hline p21 & 1.00 & 2.00 & 0.35 & 0.64 & 8.02 \\
\hline SKP2 & 1.00 & 0.90 & 0.23 & 1.09 & 1.70 \\
\hline
\end{tabular}

Figure 6

Effects of the HBx mutants on the expression of functional molecules in vitro. (A) Ectopic expression of Wt-HBx or HBx mutants affects the mRNA levels of PAI1, CDC20, P21, and SKP2 in HeLa cells. (B) Ectopic expression of Wt-HBx or HBx mutants affects the mRNA levels of PAI1, CDC20, P21, and SKP2 in HepG2 cells. (C) Ectopic expression of Wt-HBx or HBx mutants affects the protein levels of PAl1, CDC20, P21, and SKP2 in HeLa cells. (D) Ectopic expression of Wt-HBx or HBx mutants affects the protein levels of PAI1, CDC20, P21, and SKP2 in HepG2 cells. ${ }^{*} \mathrm{P}<0.05 ; * \star \mathrm{P}<0.01$; $* \star * \mathrm{P}<0.001$; ${ }^{\star \star \star \star} \mathrm{P}<0.0001$. 


\section{Figure 7}

WT

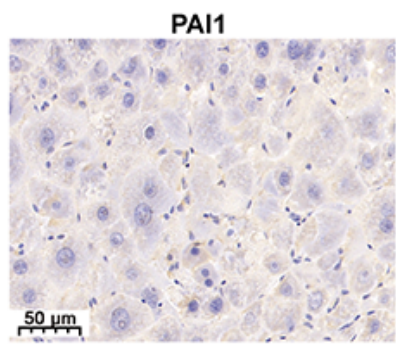

M1

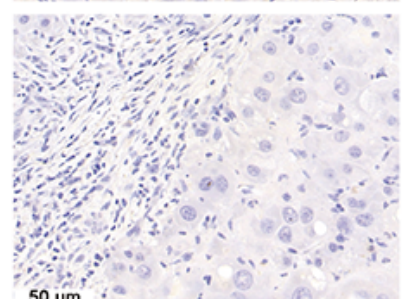

M3

$\mathrm{Ct}$

B

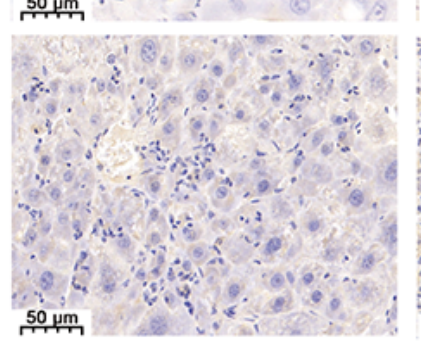

B
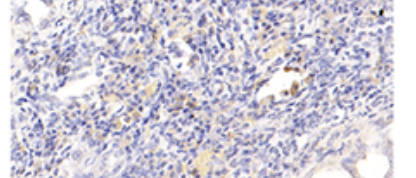

20.

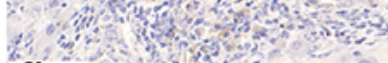

so, vm
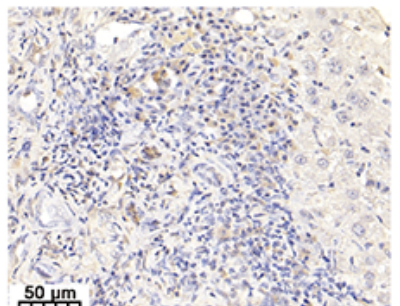

soim a dis.

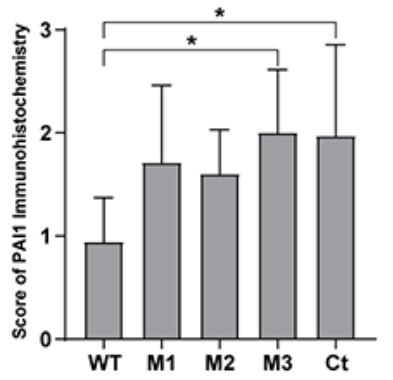

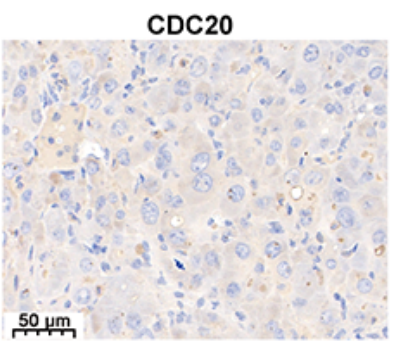
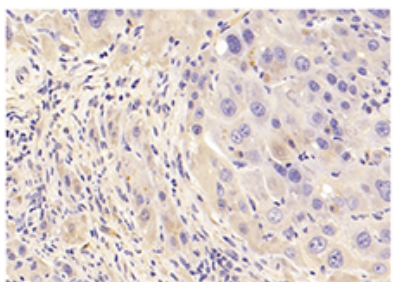

som
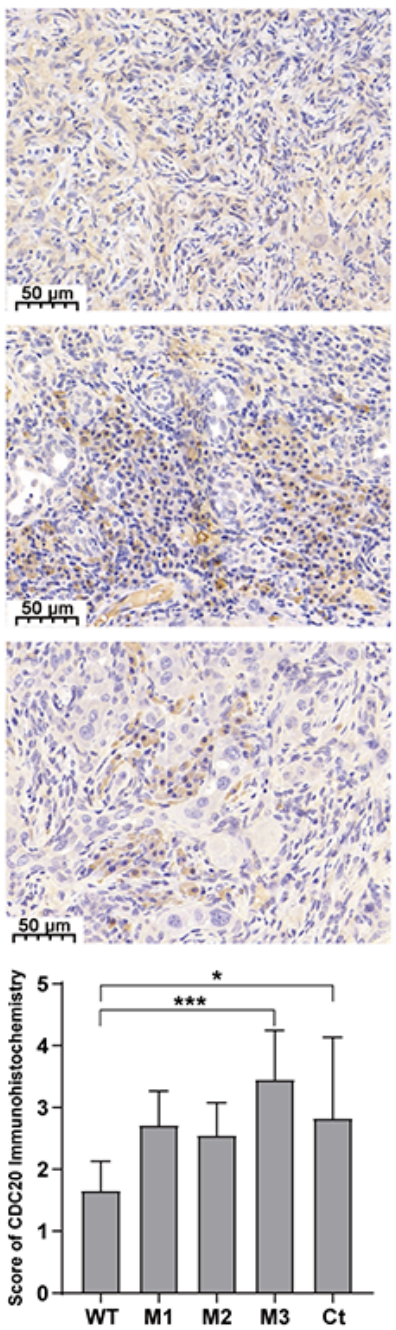
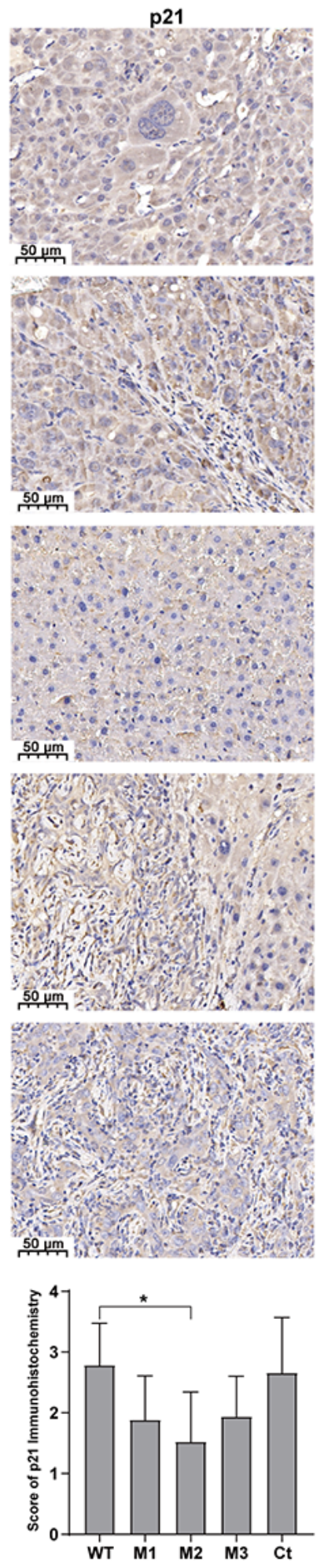

Figure 7

The expression levels of PAI 1, CDC20, and p21 in the mouse tumor tissues of the SB mouse models injected with WT-HBx or each of the four HBx mutants. (A) Representative IHC images of PAl1 staining, CDC20 staining, and p21 staining. (B) Histograms of the IHC scores for the protein expression of PAI1, CDC20, and $\mathrm{p} 21 .{ }^{*} \mathrm{P}<0.05 ; * \star * \mathrm{P}<0.001$. 
Figure 8
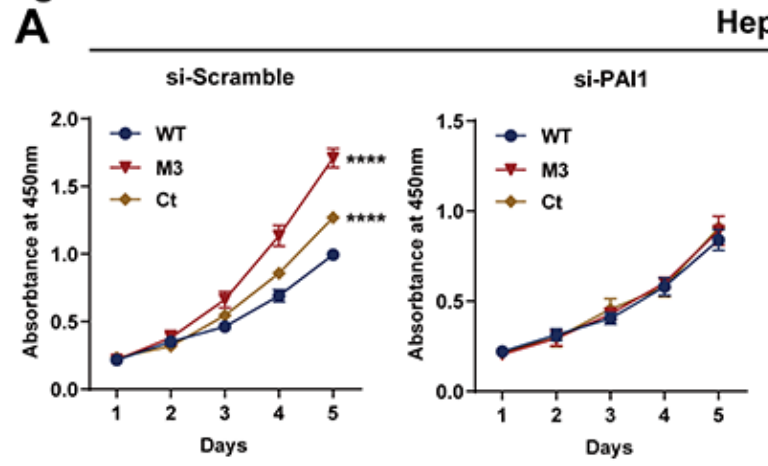

HepG2
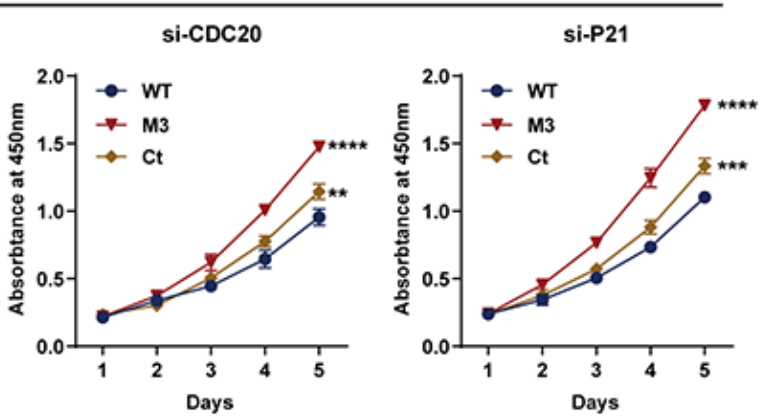

B

HeLa
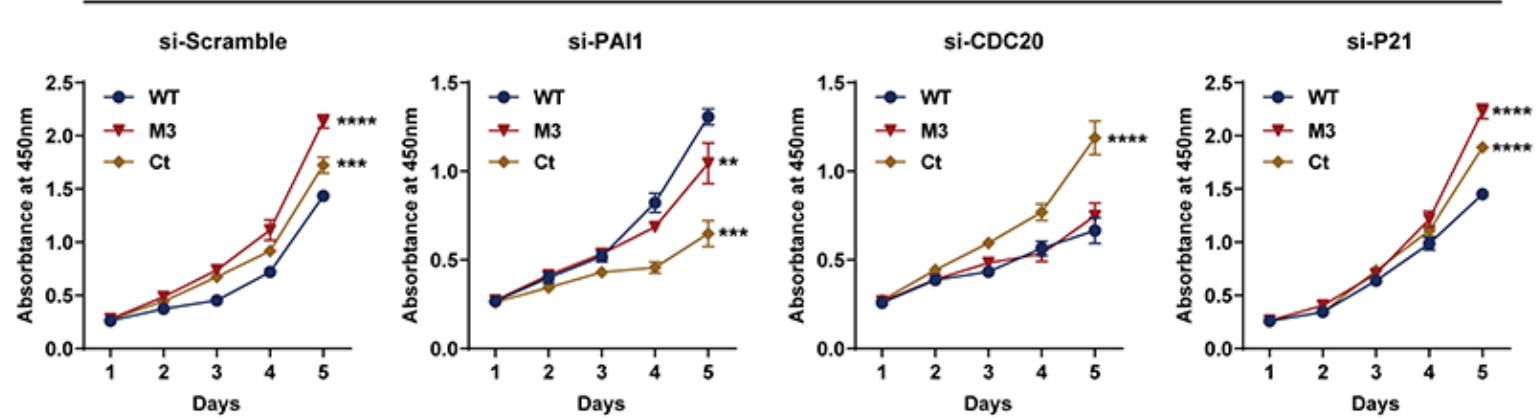

C

HepG2
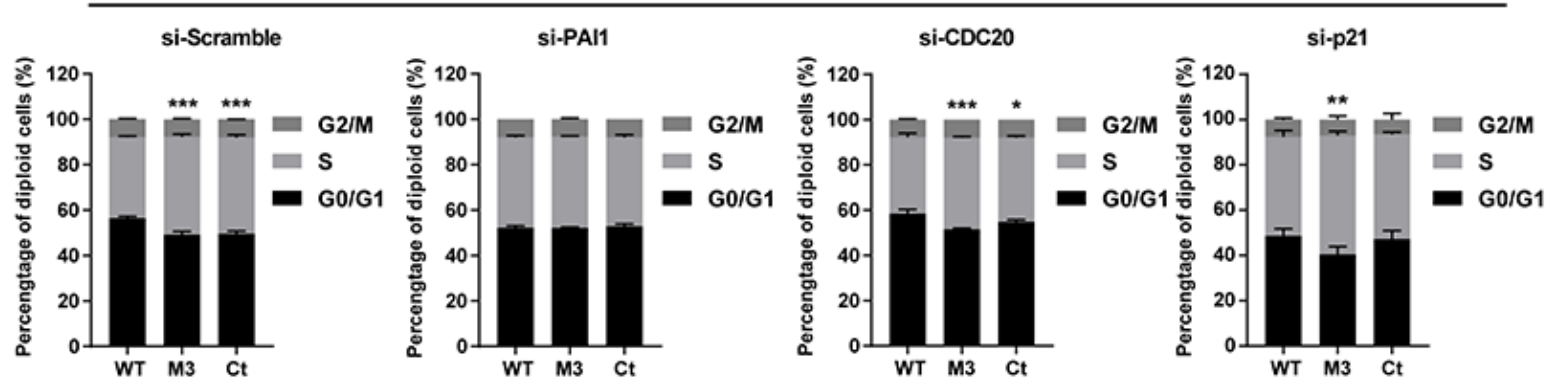

D

HeLa
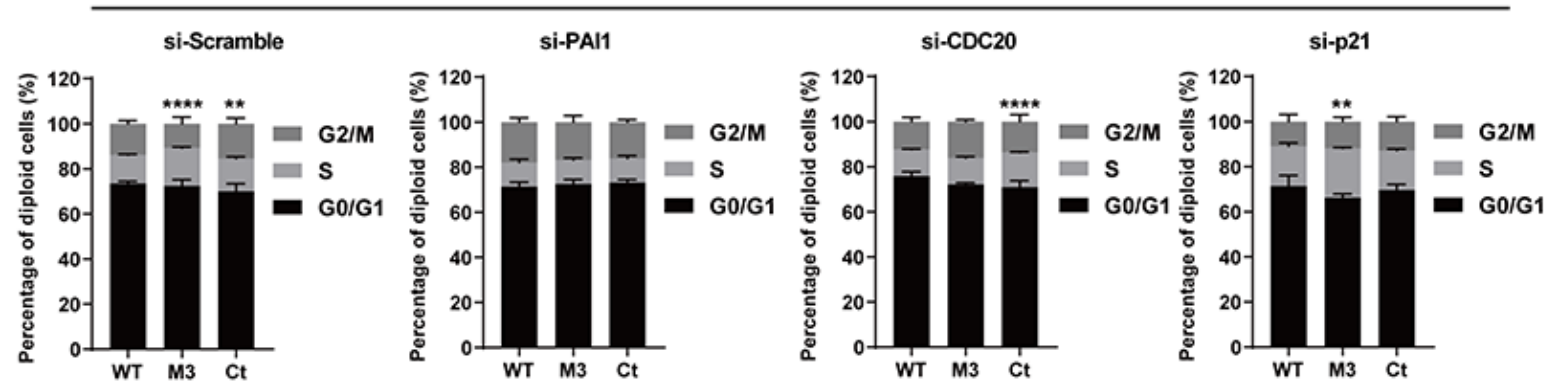

\section{Figure 8}

Silencing functional molecules influences the oncogenic effects of the HBx mutants. (A) Silencing PAl1, CDC20, and p21 influences the effects of HBx mutants on the proliferation of HepG2 cells. (B) Silencing PAI1, CDC20, and p21 influences the effects of HBx mutants on the proliferation of HeLa cells. (C) Silencing PAI1, CDC20, and p21 influences the effects of HBx mutants on the cell cycle in HepG2 cells. 
(D) Silencing PAl1, CDC20, and p21 influences the effects of HBx mutants on the cell cycle in HeLa cells. WT-HBx served as a control group in all comparisons.

\section{Supplementary Files}

This is a list of supplementary files associated with this preprint. Click to download.

- Figures1.pdf

- FigureS2.pdf

- Figures3.pdf

- FigureS4.pdf

- FigureS5.pdf

- FigureS6.pdf

- FigureS7.pdf

- Supplementorymaterial.doc

- TableS1.doc

- Tables2.doc

- TableS3.doc

- TableS4.doc

- TableS5.docx

- TableS6.docx 